\title{
Computational Assessment of a Modular Composite Wind Turbine Blade Joint
}

\author{
Norimichi Nanami ${ }^{\mathrm{a},{ }^{*}}$ and Ozden O. Ochoa ${ }^{\mathrm{b}}$ \\ ${ }^{a}$ Department of Mechanical Engineering, College of Science and Technology, Nihon University, 1-8-14, \\ Kanda-Surugadai, Chiyoda, Tokyo 101-8308, Japan \\ ${ }^{b}$ Department of Mechanical Engineering, the Dwight Look College of Engineering, Texas A\&M University, \\ 3123 TAMU, College Station, Texas, 77843-3123, USA
}

\begin{abstract}
Wind energy is one of the most promising and mature alternatives to satisfy the global demand for energy as the world population and the economic activity surge. The wind energy market has grown rapidly in the last couple of decades, boosting up the size of wind turbines to generate higher power output. Typically, the larger/longer blade designs rely on hybrid material systems such as carbon and/or glass fiber (CF/GF) reinforced polymers to improve specific stiffness/strength and damage tolerance.

Herein, we propose a computational design concept for a modular hybrid composite wind turbine blade that maintains its structural integrity and serviceability requirements. The modular configuration will simplify manufacturing-assembly processes and reduce expenses both in transportation and facilities requirements. The $80 \mathrm{~m}$ blade in this study is composed of two sections that are joined together with an innovative compression joint. Our results when compared to a single continuous blade, showed no significant alterations to its structural response. It is concluded that the proposed computational design concept that allow two modular blades to create full-length blade with robust joints is achievable. This modular concept can be easily extended for further multi-section modular blade configurations.
\end{abstract}

Keywords: Finite element analysis, Joining design concept, Structural response, Hybrid composite materials, Large-scale wind turbine blades.

\section{INTRODUCTION}

Wind energy is a natural energy resource and is a viable alternative to satisfy the future energy needs of an increasing world population and improving economic activity. It is capable of providing 72 TW (TW $=10^{12} \mathrm{~W}$ ) of electric power, which is approximately four times the world energy consumption in 2012 [1, 2]. The development of wind turbines will be a significant key factor to satisfy the prospective energy demand and to achieve a safe world. After the 2011 Fukushima nuclear disaster, the support of clean energy is now more emphatically called for the long-term global developments and government policies that may have slowed down nuclear power [3]. In order to overcome the financial hardships and create employment, the Fukushima floating offshore wind farm demonstration project was launched [4]. The size of wind turbines has grown rapidly in the last two decades, resulting in proportionally greater power output. Electrical power extracted from a horizontal-axis wind turbine (HAWT) increases with the square of a rotor radius, the cube of wind speed, and the power coefficient of a wind turbine. Recently, HAWTs with a rotor of $126-164 \mathrm{~m}$ diameter that generate power in the range of $4.0 \mathrm{MW}$ to

\footnotetext{
*Address correspondence to this author at the Department of Mechanical Engineering, College of Science and Technology, Nihon University,1-8-14, Kanda-Surugadai, Chiyoda, Tokyo 101-8308, Japan;

Tel/Fax: +81(3)3259.0735; E-mail: nnanami@mech.cst.nihon-u.ac.jp
}

8.0 MW have been installed [5-7]. The structural response and damage assessment of the $80 \mathrm{~m}$ wind turbine blade has been studied in detail by Nanami [810]. As the rotor radius (i.e., the blade length) increases, the blade weight increases exponentially. Utilization of carbon fibers in the long blade enables a thinner and more efficient blade profile along with a stiffer, slender, and lighter blade. This also results in increased material costs that dominate the overall cost of the blade for a large-size wind blade. Therefore, hybrid material systems such as carbon and glass fiber (CF/GF) reinforced composite materials are of significant attraction to the large/robust blade designs in order to improve specific stiffness/strength and damage tolerance of the blade.

In addition to the increase in the blade weight caused by the blade extension, manufacturing a largescale wind turbine blade is a significant challenge which introduces further complications in facilities and transportation. Since up to $56 \mathrm{~m}$ blade can be transported by trailer in the U.S., we will face the transportation limitation in the near future [11]. It is very desirable to develop modular construction and successful joint designs without alterations in structural integrity and stability requirements. Gamesa and Enercon have already constructed a blade in two sections connected together with a rigid metal joint $[12,13]$. A bolted joint system for sectional blades was 
developed and validated by Saenz et al. [14]. Modular space frame blade concept was proposed by Wetzel [15]. Additional concepts that explored various connectors, receptacles, threaded fasteners, or overlapped stiffeners were patented [16-18].

Herein, we computationally explore a design concept that consists of modular construction and appropriate joining techniques while adhering to the structural integrity and service ability requirements. Subsequently, the feasibility and advantages of introducing the joint concept for a modular hybrid composite wind turbine blade will be assessed with various airfoils and composite architectures for the 80 $\mathrm{m}$ blade. Our computational assessment study of the $80 \mathrm{~m}$ hybrid composite blade is undertaken in Abaqus, commercial finite element analysis (FEA) software, to demonstrate both feasibility and advantages of the present joint concept.

\section{SINGLE BLADE DESCRIPTION}

The overall geometry including airfoil construction and material selections are presented in the following sections. The blade of our 8MW wind turbine, designed to satisfy the future energy demand, has a rotor radius of $80 \mathrm{~m}$ with spar cap and shear web configuration (herein referred to as SB). The SB model is developed to understand its static and dynamic behaviors, and the blade design studies will be extended to address conceptual assembly mechanisms for a modular blade configuration discussed later.

\subsection{Geometric Specifics}

The full blade geometry in Figure $\mathbf{1}$ is modeled in Solid Works, and for simplicity, the pre-twist angle distribution is neglected in the blade model. The thickairfoil family (NREL S816, S817, S818) is employed for the blade because of its excellent aerodynamic performance [19]. The blade in this study is generated based on the following non-dimensional specifications: The span station defined as a function of $r / R$ describes the non-dimensional local rotor radius, i.e., $r / R=0$ indicates the hub center, and $r / R=1$ denotes the blade tip. The non-dimensional chord $(c / R)$ distribution along the span station is the same as what was used by Griffin $[20,21]$. The blade cross-section shape and ratio of the blade thickness to the chord length $(t / c)$ along the span station are proposed by Somers [19]. These non-dimensional specifications are summarized in Table 1, and the $80 \mathrm{~m}$ blade model for this study is extrapolated based on the specifications.

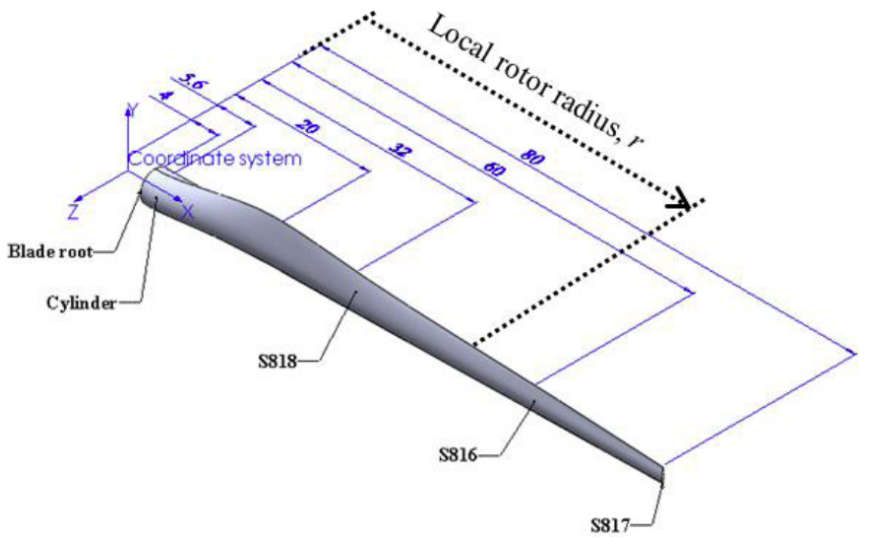

Figure 1: Dimensions of the $80 \mathrm{~m}$ blade.

Table 1: Extracted Geometric Specifications

\begin{tabular}{|c|c|c|c|}
\hline $\mathbf{r} / \mathbf{R}$ & Cross-section & $\mathbf{c / R}$ & $\mathbf{t} / \mathbf{c}$ \\
\hline \hline 0.05 & Cylinder & 0.055 & 1 \\
\hline 0.07 & Cylinder & 0.055 & 1 \\
\hline 0.25 & S818 & 0.08 & 0.24 \\
\hline 0.4 & S818 & 0.0692 & 0.24 \\
\hline 0.75 & S816 & 0.044 & 0.21 \\
\hline 1 & S817 & 0.026 & 0.16 \\
\hline
\end{tabular}

Spar cap and shear web as internal reinforcements of the blade are constructed in the airfoil section $(0.07<r / R<1)$. To improve buckling stability in the blade, the forward and aft shear web is attached at the recommended positions [20,21]. Spar cap is located between the forward and aft shear web. Dorsally the spar cap width remains constant between $r / R=0.07$ and $r / R=0.25$, and this width is linearly decreased further to the blade tip.

\subsection{Composite Layup Description}

Our selection of materials includes balsa wood, unidirectional (UD) GF, GF fabric, and CF fabric. The root section $(0.05<r / R<0.07)$ of the blade consists of GF fabric layers since GF fabric layers are economical and provide enough specific stiffness/strength to bond a metallic flange or bolt connection to the root section. In the airfoil section, the blade skin and shear web are treated as sandwich constructions of GF layers with balsa wood as a core in order to avoid the occurrence of buckling. GF fabric layers are employed for the face laminate in the skin, and the face in the webs is laminated with $0^{\circ}, 45^{\circ}$, and $-45^{\circ}$ UD-GF layers. Hybrid GF/CF composite laminates which contain 15\% CF fabric and $85 \%$ UD-GF layer reinforcement by volume 
Table 2: Thickness Distribution of the Core Material and Proposed Spar Cap in Meters

\begin{tabular}{|c|c|c|c|c|}
\hline \multirow{2}{*}{$\begin{array}{c}\text { Range of local } \\
\text { rotor radius, } \boldsymbol{r}\end{array}$} & \multicolumn{3}{|c|}{ Thicknesses of core materials } \\
\cline { 2 - 5 } & Forward blade skin & Aft blade skin & Shear web & \multirow{2}{*}{0.074} \\
\hline \hline $5.6-20$ & 0.032 & 0.054 & 0.054 & 0.036 \\
\hline $20-32$ & 0.036 & 0.060 & 0.060 & 0.029 \\
\hline $32-46$ & 0.030 & 0.050 & 0.050 & 0.022 \\
\hline $46-60$ & 0.020 & 0.044 & 0.032 & 0.016 \\
\hline $60-70$ & 0.016 & 0.035 & 0.025 & 0.012 \\
\hline $70-75$ & 0.013 & 0.029 & 0.021 & 0.018 \\
\hline $75-80$ & 0.011 & 0.025 & & 0.009 \\
\hline
\end{tabular}

are used for a spar cap. A spar cap has to provide high specific stiffness/strength and high price-performance ratio. Generally, UD fiber layers of a spar cap are used to carry bending loads while off-axis or fabric layers of a spar cap are employed to resist shear deformations. Since carbon fibers are much expensive as compared with glass fibers, it is not feasible to use only CF layers to construct a spar cap. The hybrid CF/GF spar cap laminate is proposed based on cost, stiffness, strength, and weight.

Next, we describe our design for layer thicknesses. In the root section, $40 \mathrm{~mm}$ thick laminate is employed to mitigate high stresses arising from the root connection into the hub. In sandwich laminates of the airfoil section, a constant thick face sheet of composite sandwich is used while the core thickness is assigned as a function of the chord length. The thickness of the spar cap laminate corresponds to $2.5 \%$ of the local maximum cross-section thickness. The thickness of the spar cap laminate and core material is assigned as a step function to incorporate a gradual taper in laminate thickness. Briefly, the thickness distribution in the airfoil section is presented in Table 2. As a result, the weight of the blade is calculated to be 45.8 tons.

\subsection{Design Loads}

The blade is subjected to various types of loads in operation such as aerodynamic, inertial, gravitational, and service loads as well as impact loads. Mostly aerodynamic loads such as lift and drag forces contribute to the deformation of the blade. Lift and drag forces are dependent on wind speed, and it is designed to have their maximum values in a range of wind speeds $(10-12 \mathrm{~m} / \mathrm{s})$. Lift and drag forces of an infinitesimal blade element are calculated using twodimensional airfoil characteristics, disregarding the velocity along the rotor radius and three-dimensional effects. Lift $(\delta \mathbf{L})$ and drag $(\delta \mathrm{D})$ forces for the rotating blade with an angle of attack $(\alpha)$ are considered. Resultant relative wind velocity $\left(V_{\text {rel }}\right)$ for the rotating blade is represented by Equation (1) where $u_{w}$ is wind speed, and $u_{t a}$ is a tangential air flow velocity acting in the direction opposed to the tangential velocity of the blade $\left(u_{t b}\right)[22]$.

$V_{r e l}=\sqrt{u_{w}^{2}+u_{t a}^{2}}$

Lift forces normal to a resultant relative wind velocity and drag forces parallel to a resultant relative wind velocity are given by

$\delta L / c \delta r=\rho_{\text {air }} V_{r e l}^{2} C_{l} / 2$ and $\delta D / c \delta r=\rho_{\text {air }} V_{r e l}^{2} C_{d} / 2$

Here, $\delta r$ is the infinitesimal blade length; $\rho_{\text {air }}$ an air density; $C_{l}$, a lift coefficient; $C_{d}$, a drag coefficient [22]. Air density is selected to reflect the tower height. Lift and drag coefficient corresponding to the limit angle of attack $\left(\alpha_{l}\right)$ is selected to have the upper limit of $C_{l}$ in a low-drag lift coefficient range [19]. The tangential velocity of the blade is linear speed of the rotating blade with a constant angular velocity $(\Omega)$ which varies along the rotor radius. The ratio $(\lambda)$ of tangential velocity of the blade to wind speed at $r$ is presented in Equation (3). At the blade tip (i.e., $r=R$ ), Equation (3) can be rewritten in Equation (4), called a tip speed ratio (TSR) [22, 23].

$\lambda=u_{t b} / u_{w}=r \Omega / u_{w}$

$\mathrm{TSR}=R \Omega / u_{w}$

Herein, the TSR is assumed to be a constant value, and the speed ratio linearly increases along the rotor radius. For a given wind speed and TSR value, angular velocity of the blade can be determined from Equation (4). The tangential velocity of the blade along the rotor 
radius can be obtained from Equation (3). Finally, the resultant of each decomposed load component along the blade $\mathrm{Y}$ - and Z-axis become, respectively:

$d P_{Z}=(\delta L / c \delta r) \cos \alpha_{l}+(\delta D / c \delta r) \sin \alpha_{l}$

and

$d P_{Y}=-(\delta L / c \delta r) \sin \alpha_{l}+(\delta D / c \delta r) \cos \alpha_{l}$

In addition to aerodynamic loads, inertial loads including centrifugal and gyroscopic loads are of importance for rotating blades. Since the blade is deflected in the out-of-plane direction due to aerodynamic loads, a tensile load generated by a centrifugal force is slightly deviated from the rotational plane. The tensile load acts on the blade to push back the blade on the rotational plane. This phenomenon is known as a centrifugal relief. Additionally, gyroscopic loads on the blades occur when a wind turbine yaws in operation. Gravity loading on the blade leads to an inplane bending moment that sinusoidally varies along with the position of the blade. The moment due to gravity reaches the maximum value when the blade is horizontally positioned and feathered. The service loads that result from control systems of a wind turbine such as braking and blade-pitch control act on the root section of the blade [22]. Therefore, lift, drag, and centrifugal forces only are taken into account for this study.

\section{COMPUTATIONAL SIMULATION}

\subsection{Element/Mesh Selection}

The blade of various geometric and material specifications is represented with three-dimensional linear shell elements (S3R/S4R) that follow the midplane shell formulation to avoid the influence of their torsional response [24, 25]. These elements are valid for both thin and thick shell problems and suitable for nonlinear geometrical analyses [26]. Its finite element (FE) model is created on Hyper Mesh, and the mesh size of $0.15 \mathrm{~m}$ is employed, generating 41,368 elements. The SB model is analyzed in Abaqus/Standard.

\subsection{Material Properties}

A linear elastic stress-strain constitutive relationship, the Generalized Hooke's law, is incorporated in materials. Von Mises yield criterion is employed to evaluate the initial damage of balsa wood. When the von Mises stress exceeds the allowable strength of balsa wood, balsa wood is found to be initially damaged. Balsa wood has the Young's modulus $(E)$ of $4.1 \mathrm{GPa}$, Poisson's ratio $(v)$ of 0.3 , yield strength $\left(X_{T}\right)$ of $5.4 \mathrm{MPa}$, and density $\left(\rho_{m}\right)$ of $155 \mathrm{~kg} / \mathrm{m}^{3}$ [27].

Besides, Hashin damage initiation criteria for the composites identify four different damage modes: fiber tension, fiber compression, matrix tension, and matrix compression, as presented in Equations (6) - (9) [28, 29]. Damage initiation is detected when the initiation criteria reaches the value of 1 . The post-damage initiation behavior of the composites is not considered in simulations. Thus, the stiffness degradation of the composite layer enabling progressive damage tracking is not monitored. The homogenized elastic properties based on the classical lamination theory and allowable strength of the composites are presented in Table 3 [30-32].

Table 3: Linear Elastic Properties and Allowable Strength

\begin{tabular}{|c|c|c|c|}
\hline & UD-GF & $\begin{array}{c}\text { GF fabric } \\
{[\mathbf{0} / 90]_{\mathbf{s}}}\end{array}$ & $\begin{array}{c}\text { CF fabric } \\
{[\mathbf{0} / 90]_{\mathbf{s}}}\end{array}$ \\
\hline \hline$\rho_{m}\left(\mathrm{~kg} / \mathrm{m}^{3}\right)$ & 2,100 & 2,100 & 1,600 \\
\hline$E_{1}(\mathrm{GPa})$ & 46 & 21 & 47 \\
\hline$E_{2}(\mathrm{GPa})$ & 13 & 21 & 47 \\
\hline$E_{3}(\mathrm{GPa})$ & 13 & 8.55 & 10 \\
\hline$G_{12}(\mathrm{GPa})$ & 5 & 3.7 & 3.78 \\
\hline$G_{13}(\mathrm{GPa})$ & 5 & 3.5 & 3.5 \\
\hline$G_{23}(\mathrm{GPa})$ & 4.6 & 3.5 & 3.5 \\
\hline$V_{12}$ & 0.3 & 0.183 & 0.33 \\
\hline$V_{13}$ & 0.3 & 0.0305 & 0.33 \\
\hline$V_{23}$ & 0.42 & 0.075 & 0.07 \\
\hline$X_{T} / X_{C}(\mathrm{MPa})$ & $1,080 / 620$ & $367 / 549$ & $627 / 572$ \\
\hline$Y_{T} / Y_{C}(\mathrm{MPa})$ & $39 / 128$ & $367 / 549$ & $627 / 572$ \\
\hline$S_{L} / S_{T}(\mathrm{MPa})$ & $89 / 64$ & $97.1 / 274.5$ & $80 / 286$ \\
\hline
\end{tabular}

If $\hat{\sigma} \geq 0$, fiber tension mode:

$\left(\hat{\sigma}_{11} / X_{T}\right)^{2}+\alpha_{c}\left(\tau_{12} / S_{L}\right)^{2} \geq 1$

If $\hat{\sigma}_{11}<0$, fiber tension mode:

$\left(\hat{\sigma}_{11} / X_{C}\right)^{2} \geq 1$

If $\hat{\sigma}_{22} \geq 0$, matrix tension mode:

$\left(\hat{\sigma}_{22} / Y_{T}\right)^{2}+\left(\hat{\tau}_{12} / S_{L}\right)^{2} \geq 1$ 
If $\hat{\sigma}_{22}<0$, matrix compression mode:

$$
\left(\hat{\sigma}_{22} / 2 S_{T}\right)^{2}+\left[\left(Y_{C} / 2 S_{T}\right)^{2}-1\right]\left(\hat{\sigma}_{22} / Y_{C}\right)+\left(\hat{\tau}_{12} / S_{L}\right)^{2} \geq 1
$$

In the above equations, $X_{T}$ denotes the longitudinal tensile strength; $X_{C}$, the longitudinal compressive strength; $Y_{T}$, the transverse tensile strength; $Y_{C}$, the transverse compressive strength; $S_{L}$, the longitudinal shear strength; $S_{T}$, the transverse shear strength; $\alpha_{c}$, a coefficient to determine the contribution of the shear stress to the fiber tension mode. The fiber tension mode can be specified by using either the proposed model by setting $\alpha_{C}=0$ and $S_{T}=Y_{C} / 2$ or the model with $\alpha_{c}=1[28,29]$.

\subsection{Analysis Conditions}

\subsubsection{Static Analysis}

The three rotations and three displacements are constrained at the blade root; the $\mathrm{Z}$ component of the resultant forces $\left(d P_{Z}\right)$ is applied to the lower shell surface of the blade; the $Y$ component of the resultant forces $\left(d P_{Y}\right)$ is applied both to the lower and upper shell surfaces, as shown in Figure 2.

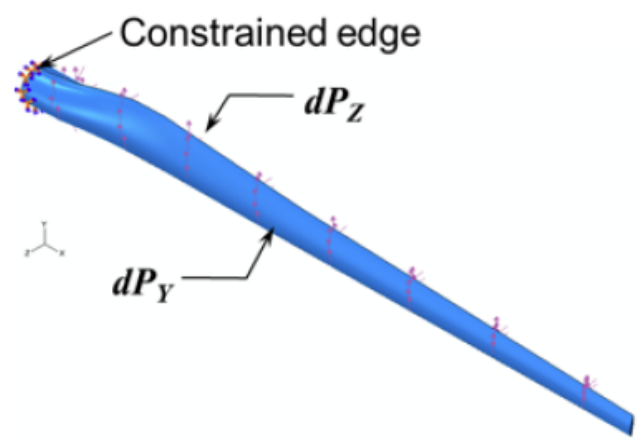

Air density is assigned as $1.208 \mathrm{~kg} / \mathrm{m}^{3}$ for the hub height. The TSR of the blade is assumed to be constant at 7 [23]. The maximum lift and drag forces associated with $u_{w}=12 \mathrm{~m} / \mathrm{s}$ are considered. Resultant relative wind speed along the local rotor radius can be found to calculate lift and drag forces. Since tangential velocity of the blade is not constant along the rotor radius, lift and drag forces are evaluated at nine locations (Nodes), creating eight sections [8]. Afterwards, lift and drag forces are decomposed into the $Y$ - and Z-axis components, $d P_{Y}$ and $d P_{Z}$, that are calculated in Equation (5) and presented in Table 4. In each section, the corresponding nodal forces are averaged to obtain the resultant forces that are distributed as step functions. Note that values of $d P_{Y}$ in Table 4 are one-half of $d P_{Y}$ values calculated in Equation (5b) since the $d P_{Y}$ is applied to two surfaces of the blade. Furthermore, the angular velocity $\left(\omega_{z}\right)$ of $1.05 \mathrm{rad} / \mathrm{s}$ about the Z-axis, generated by $u_{w}=12 \mathrm{~m} / \mathrm{s}$, is assigned to account for centrifugal forces.

\subsubsection{Frequency Analysis}

Generally, a wind turbine blade has three modes of natural frequencies: flapping, edge, and torsion. For frequency analysis, the three rotations and three

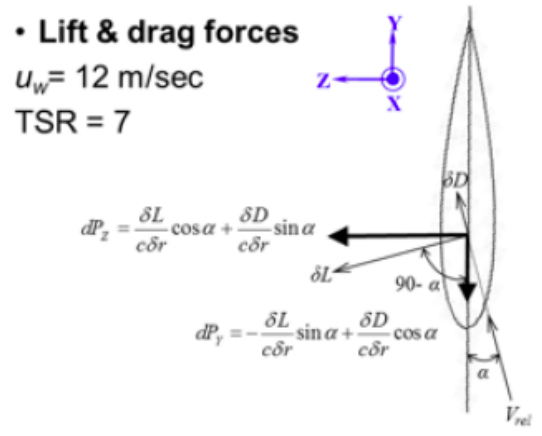

Figure 2: Boundary and loading conditions.

Table 4: Loads at Nine Nodes along the Rotor Radius

\begin{tabular}{|c|c|c|c|c|c|c|c|}
\hline Node No. & $r(m)$ & $V_{\text {rel }}(\mathrm{m} / \mathrm{s})$ & $\alpha_{1}\left(\left(^{\circ}\right)\right.$ & $c_{l}$ & $C_{d}$ & $d P_{Y}(\mathrm{~Pa})$ & $d P_{Z}(\mathrm{~Pa})$ \\
\hline 1 & 4 & 12.7 & 6.5 & 0 & 0.3 & 14.6 & 3.32 \\
\hline 2 & 5.6 & 13.4 & 6.5 & 0 & 0.3 & 16.1 & 3.66 \\
\hline 3 & 20 & 24.2 & 6.5 & 1.2 & 0.012 & -21.9 & 422 \\
\hline 4 & 32 & 35.7 & 6.5 & 1.2 & 0.012 & -47.6 & 918 \\
\hline 5 & 46 & 49.8 & 7 & 1 & 0.008 & -85.2 & 1,486 \\
\hline 6 & 60 & 64.1 & 7 & 1 & 0.008 & -142 & 2,468 \\
\hline 7 & 70 & 74.5 & 5 & 0.9 & 0.007 & -120 & 3,006 \\
\hline 8 & 75 & 79.7 & 5 & 0.9 & 0.007 & -137 & 3,439 \\
\hline 9 & 80 & 84.9 & 5 & 0.9 & 0.007 & -155 & 3,902 \\
\hline
\end{tabular}


displacements are constrained at the blade root. The natural frequencies and corresponding mode shapes are obtained for undamped and non-rotational blades. Linear perturbation scheme and Lanczos method are utilized to extend eigenvalues.

\section{MODULAR COMPOSITE BLADE DESCRIPTION}

Herein, a modular blade design is introduced to facilitate simpler manufacturing and assembly processes without handicapping the integrity and structural response of a full single blade. Conceptual assembly mechanisms for multi-section configuration of the blade are addressed to assure that the displacements, stresses, strains, and vibration modes within design and safety limits. Thus, the singlecontinuous blade (SB Model) is taken as the baseline in this study.

\subsection{The Compression Joint Concept}

In case of connecting two tubular modules without a major penalty in mass, the tubular joint concept, as presented in Figure $\mathbf{3}$, has been developed through discussions with Schneider [Schneider W, oral communication, Mechanical Engineering, Texas A\&M University: 2011 May 19]. In this concept, an inboard module is connected to an outboard module via a simple cable and plate. Compressive forces caused by pulling the cable through the plate are applied at the contact surfaces between the inboard and outboard module. Thus, two modules are rigidly kept in place due to compression. Adhesives may be used to further enhance this joint.

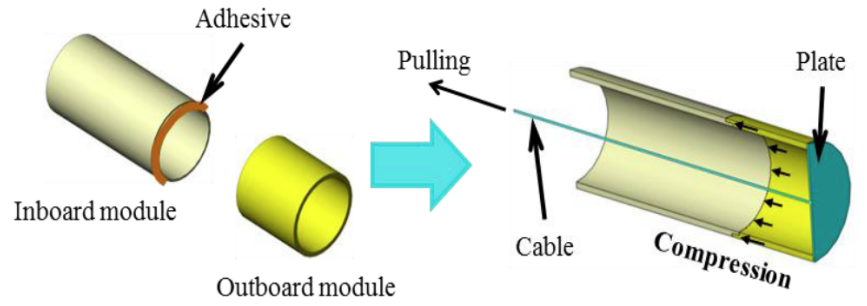

Figure 3: Tubular joint concept.

In Figure 3, a plate is used to apply axial compressive forces to a joint region so that two modules are connected rigidly. However, a shock absorber, like a rubber and spring/damper, may be inserted between a plate and an outboard module to avoid crush/damage at the contact face. The alternative approach to introduce compression to a joint region is tensile cable mechanisms employed for architectural and structural purposes such as cable- stayed, suspended cable, and turnbuckle cable systems. Also, cable mechanisms in a joint region are dependent on the magnitude of the required compressive force.

The schematic of a cantilever with one joint based on the tubular joint concept is depicted in Figure $\mathbf{4 a}$. The left end of the cantilever is fixed while the right end is free. The cantilever is subjected to bending and centrifugal forces. The free body diagram of the inboard module at the joint region is presented in Figure $\mathbf{4 b}$ where $M$ is a moment generated by bending; $Q$, a shear force generated by bending; $F_{c}$, a centrifugal force; $F$, an axial force due to cable mechanisms.

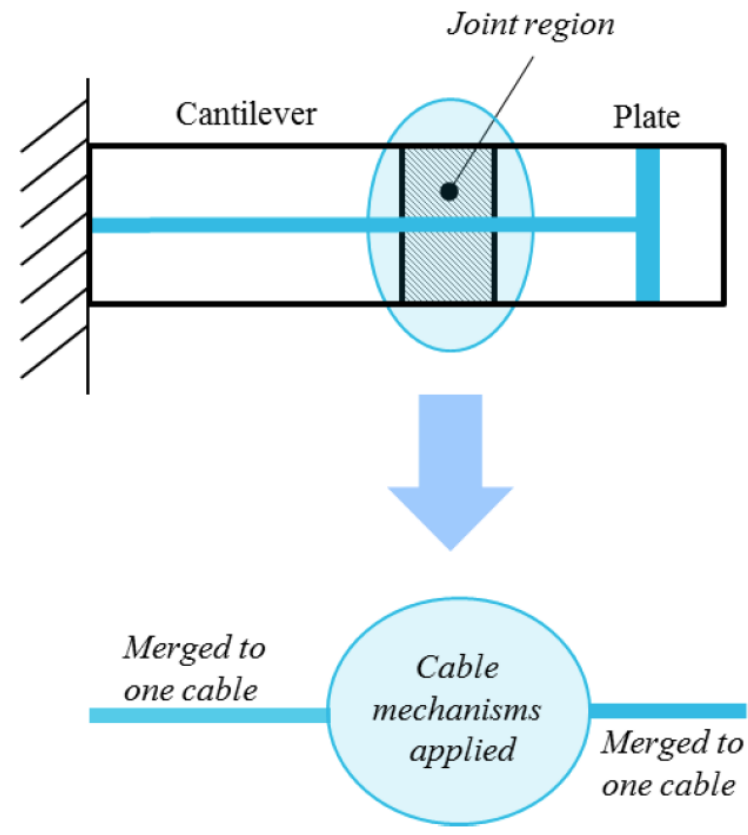

(a)

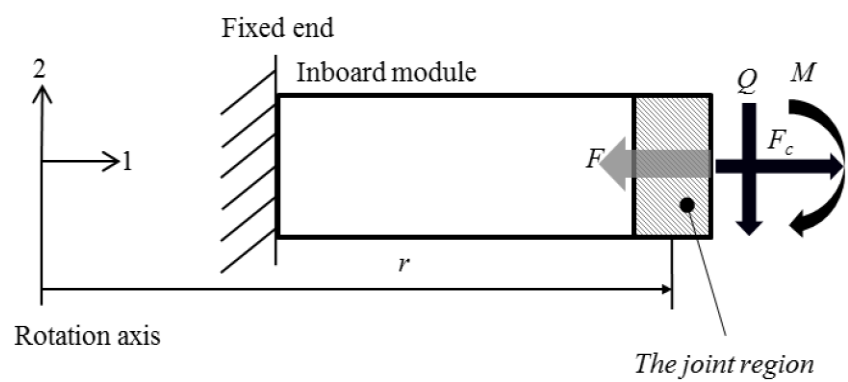

(b)

Figure 4: The tubular joint concept applied for a cantilever: (a) schematic, and (b) free body diagram.

The axial force sustained by cable mechanisms has to follow the following condition:

$A_{c} \sigma_{11}-F_{c} \leq 0$ 
where $A_{c}$ is a cross-sectional area; $\sigma_{11}$, a stress due to bending/centrifugal forces. The stress can be calculated in the equation shown below.

$\sigma_{11}=M c / I+F_{c} / A_{c}$

where $c$ is the perpendicular distance to the neutral axis; I, an area moment of inertia. Thus, the applied axial force of cable mechanisms has to be greater than the summation of stresses along the 1-direction over a cross-sectional area. The worst case scenario of stress states in a joint region has to be considered to determine the magnitude of an axial force for cable mechanisms. Thus, the maximum magnitude of $\sigma_{11}$ in a joint region is taken, and this value multiplied by the area of a cross-section yields to the required value of an axial force for cable mechanisms.

Candidate cables for cable mechanism are specialpurpose steel wires that are primarily employed in aircrafts and bridge construction [33, 34]. The steel wires are able to provide high load capacity, high strength, excellent corrosion and temperature resistance, and non-magnetic characteristic. Additionally, carbon fiber composite cables (CFCCs) are potentially adopted as a substitute for the steel wires since CFCCs provide higher specific strength/stiffness, better corrosion resistance, and better fatigue performance in comparison with the steel cables [35, 36]. These cables can be supplied from commercial vendors, and the specifications of the cables are presented in Table $\mathbf{5}$. When the required axial force for cable mechanisms is greater than the breaking strength of a single cable, using multiple cables or increasing a diameter of a single cable may be necessary to sustain the required axial force.

\subsection{Computational Modular Blade Representation}

The compression joint concept as applied to create the $80 \mathrm{~m}$ blade is depicted schematically in Figure $\mathbf{5}$. Physical geometries of the inboard and outboard modules seen in Figure $\mathbf{5 a}$ are respectively defined as follows; the inboard module extends from $r=4 \mathrm{~m}$ to $r=$ $40.05 \mathrm{~m}$, whereas the outboard module is represented as the section $37.95 \mathrm{~m}<r<80 \mathrm{~m}$. The projection of the inboard module is inserted inside the outboard module; thus, the projection is overlapped with the outboard module, creating a joint region of the blade with a length of $2.1 \mathrm{~m}$.

To create the FE modular blade model, the jointtransition-region is placed at $32 \mathrm{~m}<r<46 \mathrm{~m}$ as presented in Figure 5a. For clarity of visualization, the upper blade skin in Figure $\mathbf{5 b}$ and $\mathbf{c}$ is hidden, and FBS, BS/SC, ABS, and SW denote the forward blade skin, the blade skin/spar cap, the aft blade skin, and the shear web, respectively. It is assumed that the reinforcement region between the inboard module and outboard module are perfectly bonded at $r=37.95 \mathrm{~m}$ in this study. Furthermore, this region is computationally described as a single surface. Therefore, the jointreinforcement section marked by gray solid lines in Figure $\mathbf{5 b}$ is defined as $36.9 \mathrm{~m}<r<41.10 \mathrm{~m}$. As seen in Figure 5c, the overlapping region surrounded with red solid lines is depicted as Joint-SW and Joint-BS/SC, occupying $37.95 \mathrm{~m}<r<40.05 \mathrm{~m}$. The reinforced laminates are employed for the regions such as Reinf.FBS, Reinf.-ABS, Reinf.-SW, and Reinf.-BS/SC surrounding the overlapping region. Reinf.-FBS and Reinf.-ABS are located between $36.9 \mathrm{~m}<r<41.1 \mathrm{~m}$ (4.2 $\mathrm{m}$ in length), whereas Reinf.-SW and Reinf.-BS/SC are located at $36.9 \mathrm{~m}<r<37.95 \mathrm{~m}$ (1.05 m in length), and at $40.05 \mathrm{~m}<r<41.10 \mathrm{~m}$ (1.05 $\mathrm{m}$ in length).

Table 5: Specifications of Stainless Cables and CFCCs

\begin{tabular}{|c|c|c|c|c|c|}
\hline & $\begin{array}{l}\text { Diameter } \\
(\mathrm{m})\end{array}$ & Construction & Cross-section & $\begin{array}{l}\text { Breaking strength } \\
\text { (N) }\end{array}$ & $\begin{array}{l}\text { Unit mass } \\
(\mathrm{kg} / \mathrm{m})\end{array}$ \\
\hline & 0.04 & & & $9.11 \times 10^{5}$ & 6.46 \\
\hline $\begin{array}{c}\text { Stainless cables } \\
{[34]}\end{array}$ & 0.06 & IWRC $6 \times$ WS(36) & 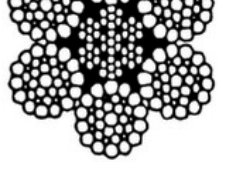 & $1.92 \times 10^{6}$ & 14.5 \\
\hline CFCCs [35] & 0.04 & $1 \times 37$ & & $1.2 \times 10^{6}$ & 1.53 \\
\hline
\end{tabular}




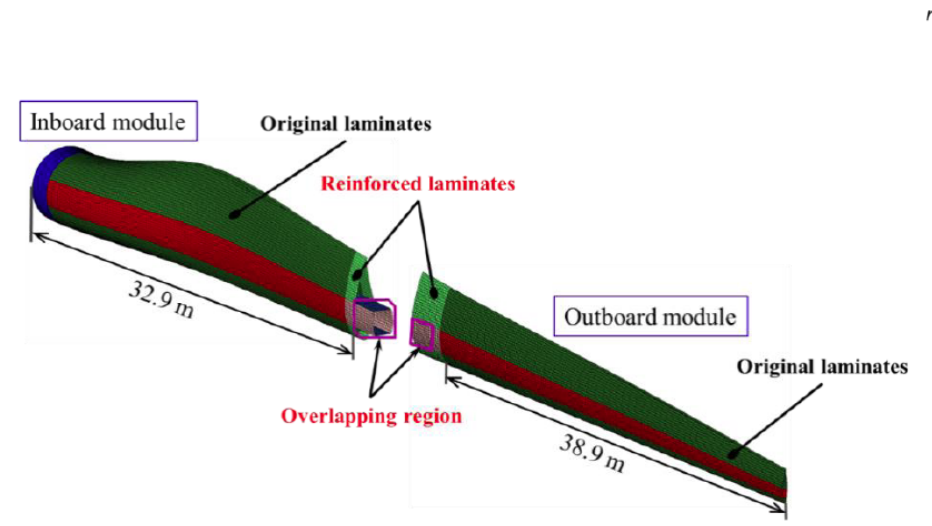

(a)

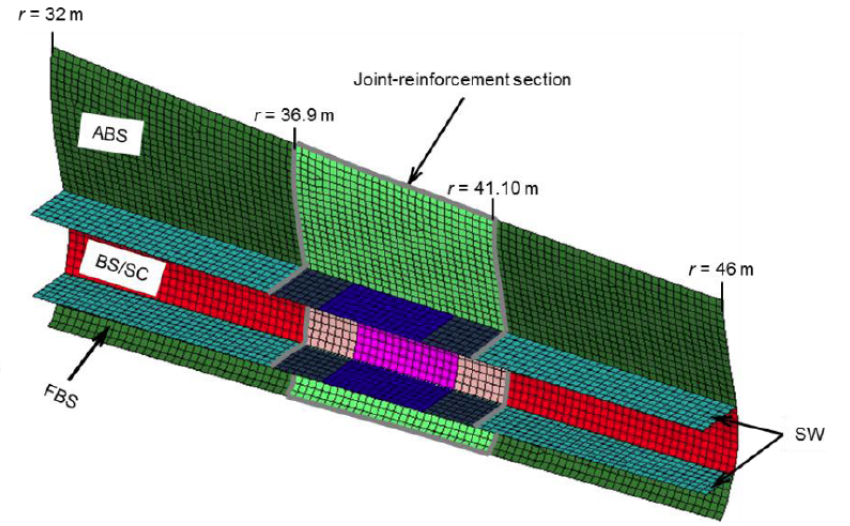

(b)

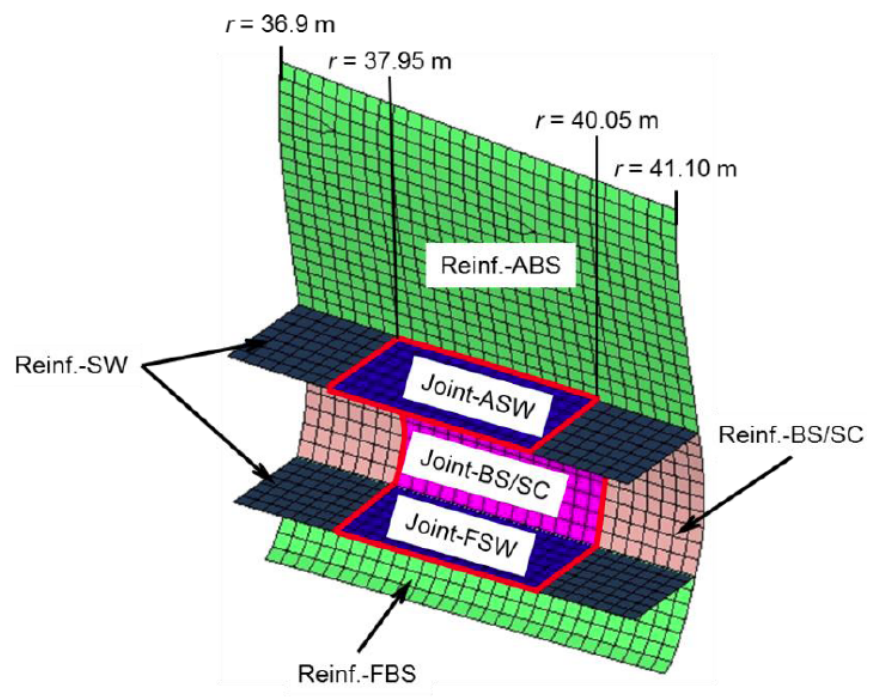

(c)

Figure 5: The conceptual schematic of the modular blade: (a) inboard and outboard modules, (b) joint-transition-region (32 m $<r<46 \mathrm{~m})$, and (c) joint-reinforcement section $(36.9 \mathrm{~m}<r<41.1 \mathrm{~m})$.

For the modular blade (MB) model, elastic moduli and shear moduli of UD-GF, GF fabric, CF fabric, and balsa wood in the joint and reinforcement regions are amplified by $3 \mathrm{X}$ and $1.5 \mathrm{X}$ of their typical values utilized in the SB Model. These values are presented in Table 6 with the following nomenclature: $E_{F B S}$, the stiffness of the forward blade skin laminate; $E_{B S / S C}$, the stiffness of the blade skin/spar cap laminate; $E_{A B S}$, the stiffness of the aft blade skin laminate; $E_{S W}$, the stiffness of the shear web laminate. The rest of all geometry, material details, and elements/meshes in the MB Model are maintained to the SB Model.

To assess the effect of increased mass in the jointreinforcement section to natural frequencies, two types of modular blade models are considered; MB Model and MB-IW Model. It is assumed that the mass change of the joint-reinforcement section is not presented in the MB Model. In the MB-IW Model, the density of materials used in the joint and reinforcement are considered to increase by $20 \%$ and $10 \%$, respectively. Then, the weight of the MB-IW Model is 46.0 tons.

\section{Results and discussion}

\subsection{Analyses Description}

The single-continuous blade (SB Model) and the modular blade with one joint (MB Model) are analyzed in Abaqus/Standard for both static and natural frequency simulations. The three rotations and displacements are fully constrained at the blade root, and the blade is subjected to aerodynamic and centrifugal forces as operational loads.

\subsection{Displacements}

The selected nodes and the corresponding tip displacements are shown in Table 7. All results presented below are obtained at the loads associated with a certain wind speed where the von Mises stress 
Table 6: Laminate Stiffness in the Joint-Transition-Region

\begin{tabular}{|c|c|c|c|c|c|}
\hline \multirow{2}{*}{$\begin{array}{l}\text { Range of } r \\
(\mathrm{~m})\end{array}$} & \multirow{2}{*}{$\begin{array}{l}\text { Length } \\
\text { (m) }\end{array}$} & \multicolumn{4}{|c|}{ Stiffness of laminates } \\
\hline & & Forward blade skin & Blade skin/spar cap & Aft blade skin & Shear web \\
\hline $32-36.9$ & 4.9 & $E_{F B S}$ & $E_{B S / S C}$ & $E_{A B S}$ & $E_{S W}$ \\
\hline $36.9-37.95$ & 1.05 & \multirow{3}{*}{$1.5 \times E_{F B S}$} & $1.5 \times E_{B S / S C}$ & \multirow{3}{*}{$1.5 \times E_{A B S}$} & $1.5 \times E_{S W}$ \\
\hline $37.95-40.05$ & 2.1 & & $3 \times E_{B S / S C}$ & & $3 \times E_{S W}$ \\
\hline $40.05-41.10$ & 1.05 & & $1.5 \times E_{B S / S C}$ & & $1.5 \times E_{S W}$ \\
\hline $41.10-46$ & 4.9 & $E_{F B S}$ & $E_{B S / S C}$ & $E_{A B S}$ & $E_{S W}$ \\
\hline
\end{tabular}

of the core exceeds its allowable. Load-I and Load-II in Table 7 represent lift, drag, and centrifugal forces, and lift and drag forces, respectively. Note that the result of Load-II is utilized to investigate the effect of centrifugal forces on displacements.

Under Load-I, $U_{2}$ displacements of the MB Model are reduced by $3 \%$ from the SB Model. However, the $\mathrm{U}_{3}$ displacement is the greatest component for both models. The joint-reinforcement section of the MB Model displays only $3 \%$ reduction in the $U_{3}$ displacement in comparison to the SB Model. It is seen in Table 7 that the tip displacements of SB Model under Load-I at $u_{\mathrm{w}}=9.3 \mathrm{~m} / \mathrm{s}$ are equivalent to those under Load-II at $u_{\mathrm{w}}=8.9 \mathrm{~m} / \mathrm{s}$. Though the SB blade becomes stiffer due to a centrifugal force, the strong phenomena of a centrifugal relief is not observed.

The global $U_{3}$ displacement contours of the upper blade skin in the region (32 $\mathrm{m}<r<46 \mathrm{~m}$ ), which has the joint-reinforcement section in the MB Model, are presented in Figure 6 to further illustrate that the modular blade as designed remains unchanged from the SB Model response.

\subsection{Stresses}

The stresses in the GF and CF layers are very small in comparison to their allowable strength in the SB Model and MB Model. The following observations are made on $S_{11}$ stress contour which is the largest component in the stress field. The asymmetric airfoil cross-section leads to higher $S_{11}$ stresses in the upper blade skin than the lower blade skin. The highest compressive stress in the outermost GF fabric layer of the upper skin for the SB Model and MB Model is 44.1 $\mathrm{MPa}$ and 44.3 MPa, respectively, as shown in Figure 7. Note that all results presented in this section are obtained under Load-I. These values occur at the blade skin/spar cap $(20 \mathrm{~m} \quad<r<32 \mathrm{~m})$ where the corresponding reaction moment is higher, but the section modulus is lower. In both models, the stresses are much lower for the rest of the airfoil section. At any given cross-section, lower stresses are seen at the

Table 7: Tip Displacements of the SB Models and MB Model

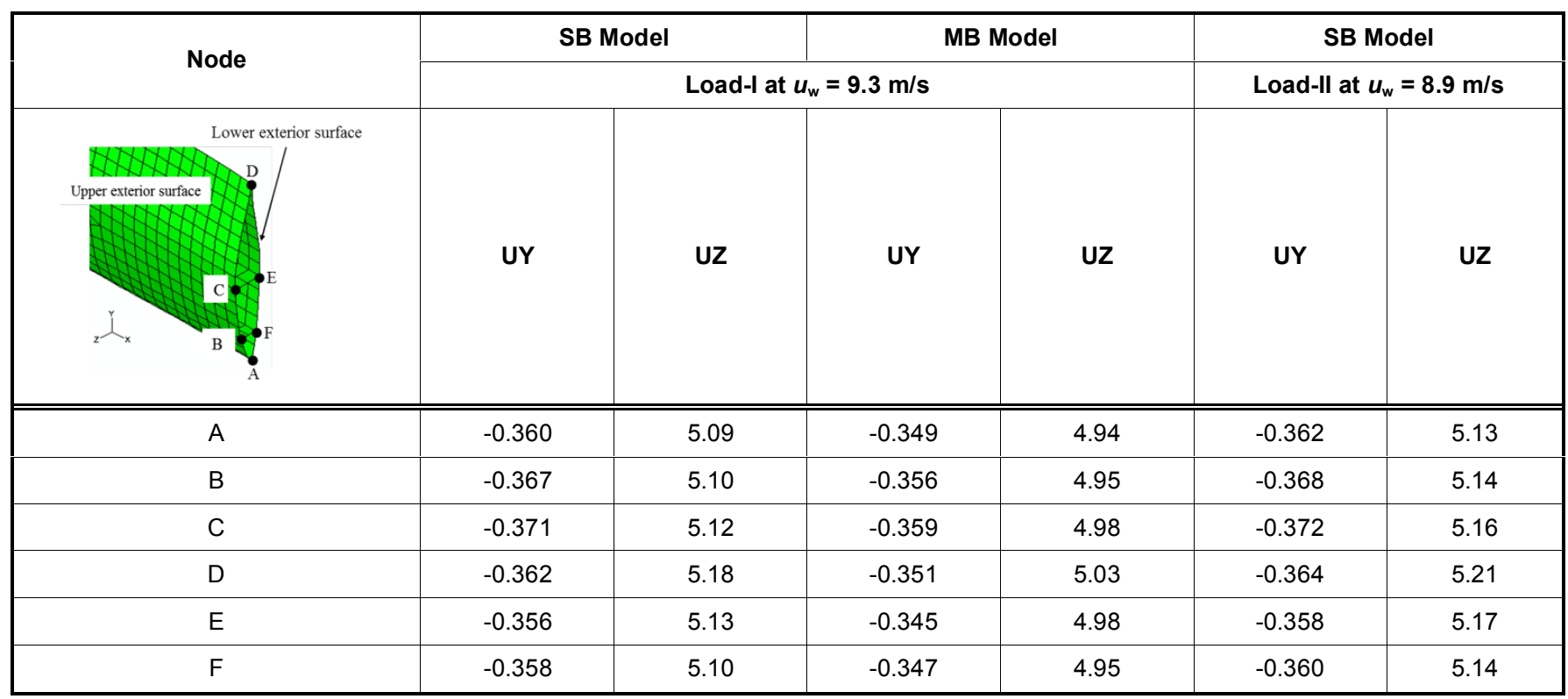



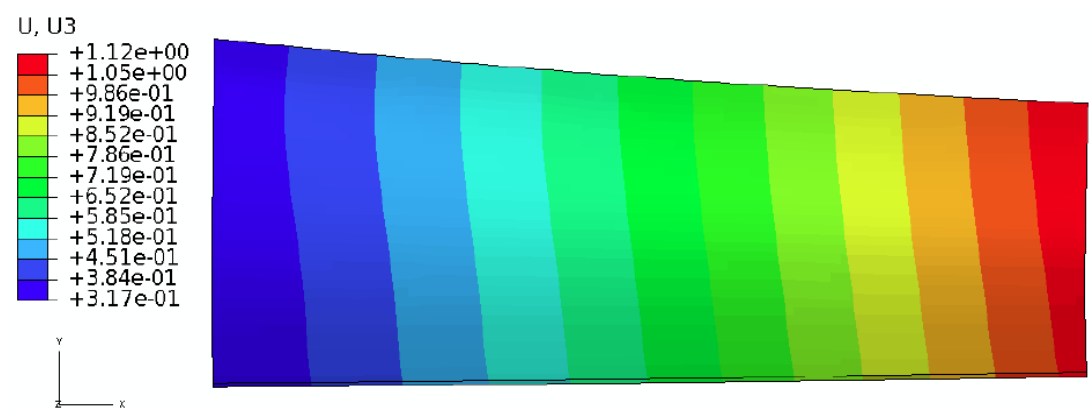

(a)

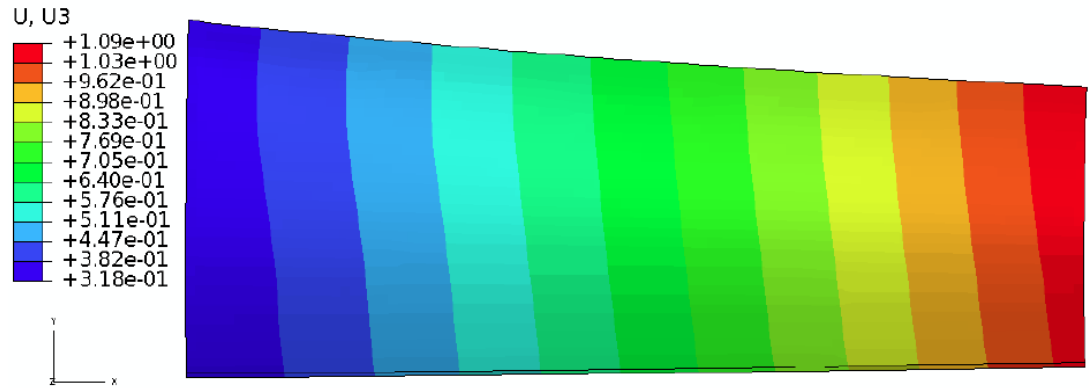

(b)

Figure 6: Global $U_{3}$ displacement contours of the upper blade skin, $32 \mathrm{~m}<r<46 \mathrm{~m}$ : (a) SB Model, and (b) MB Model.

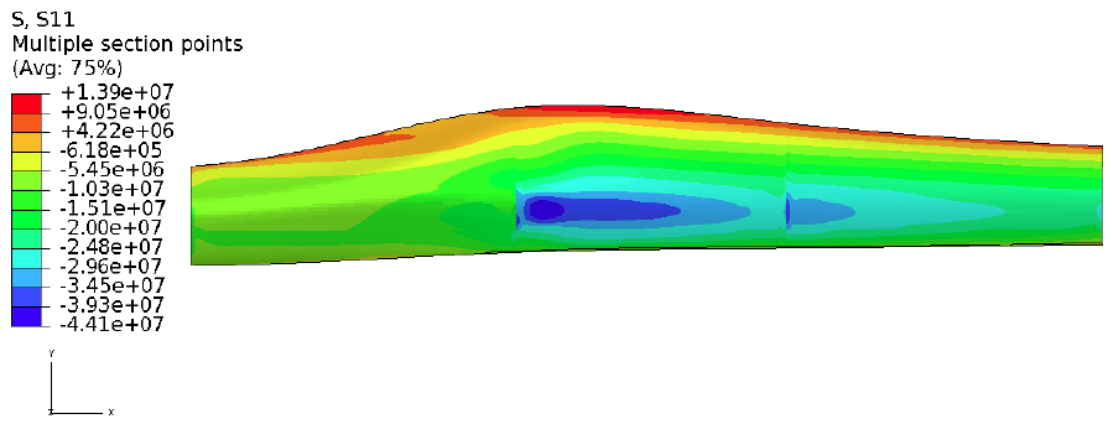

(a)

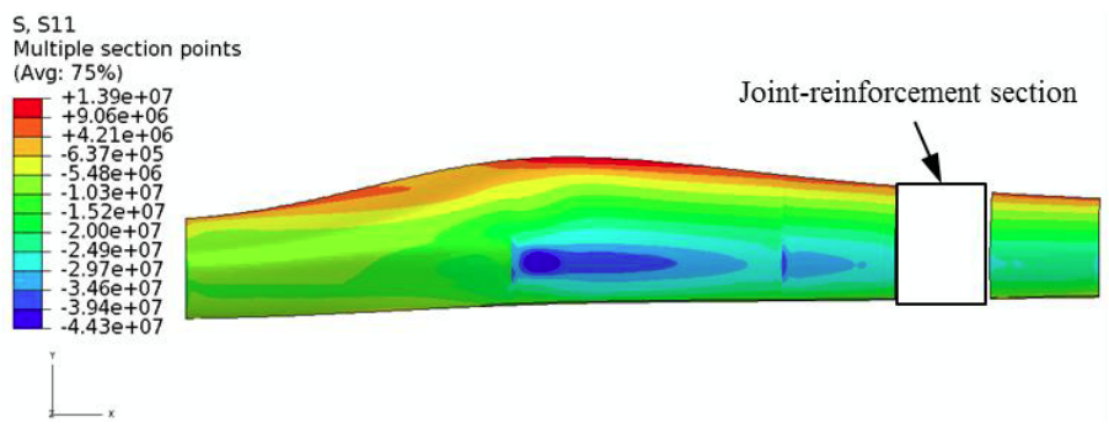

(b)

Figure 7: $\mathrm{S}_{11}$ stress in the outermost GF fabric layer in the upper blade skin $(5.6 \mathrm{~m}<r<46 \mathrm{~m})$ : (a) SB Model, and (b) MB Model.

leading or trailing edges. The compressive stresses decrease along the local rotor radius toward the tip though tensile stresses are observed in the vicinity of the tip due to asymmetric blade geometry, as depicted in Figure 8. All the other layers experience very similar contours. Thus, it is inferred that the global stress field remains almost identical between the single blade and modular blade.

To better understand the influence of the jointtransition region on the stress distribution, $S_{11}$ stress contours in the outermost GF fabric layer of the upper 


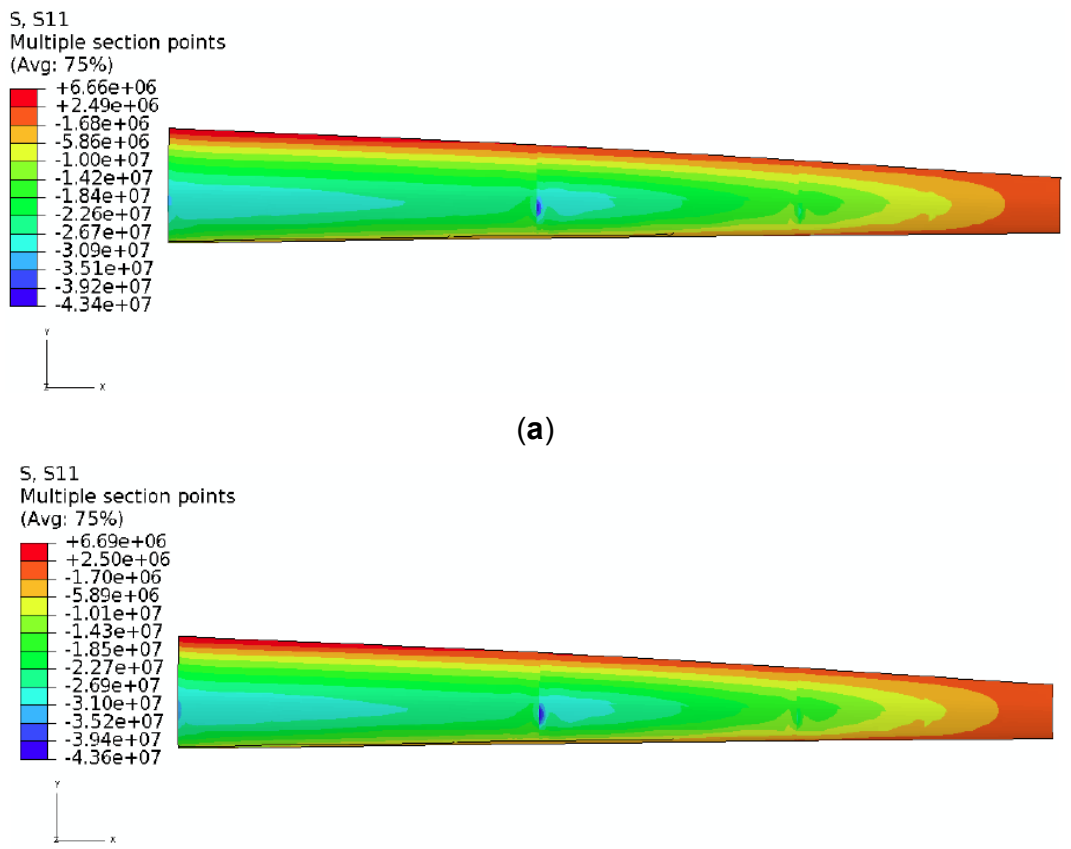

(b)

Figure 8: $\mathrm{S}_{11}$ stress in the outermost GF fabric layer in the upper blade skin $(46 \mathrm{~m}<r<80 \mathrm{~m})$ : (a) SB Model, and (b) MB Model.
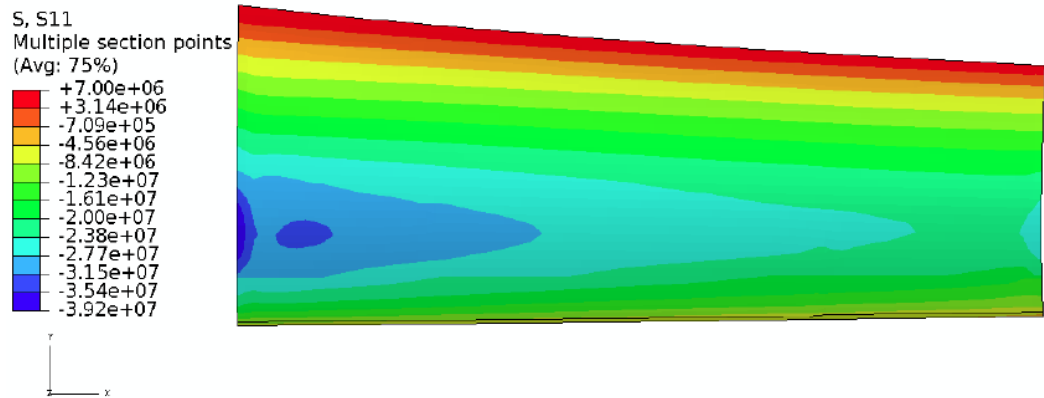

(a)

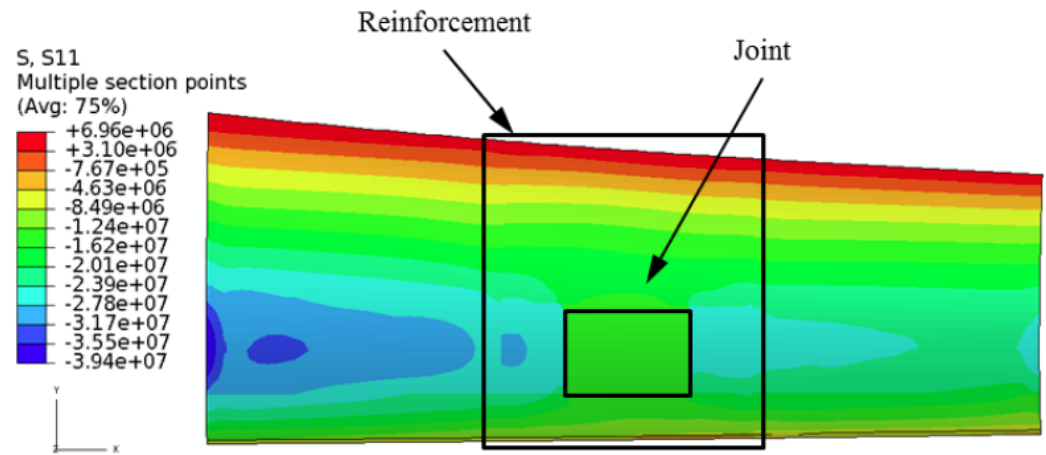

(b)

Figure 9: $\mathrm{S}_{11}$ stress in the outermost GF fabric layer in the upper blade skin $(32 \mathrm{~m}<r<46 \mathrm{~m})$ : (a) SB Model, and (b) MB Model.

blade skin (32 $\mathrm{m}<r<46)$ are presented in Figure 9. It is noted that the reinforcement region does not induce any significant changes due to its higher stiffness of 1.5x the SB Model. On the other hand, the joint region in the MB Model undergoes compressive stresses in the range of 12.4-14.8 MPa whereas in the SB Model, this region experiences 22.2-27.1 MPa in compression. This almost $45 \%$ difference is attributed to the $3 x$ higher stiffness of this local region in the MB model.

While no damage modes are observed in GF and CF layers, the balsa core of the skin in both the SB 


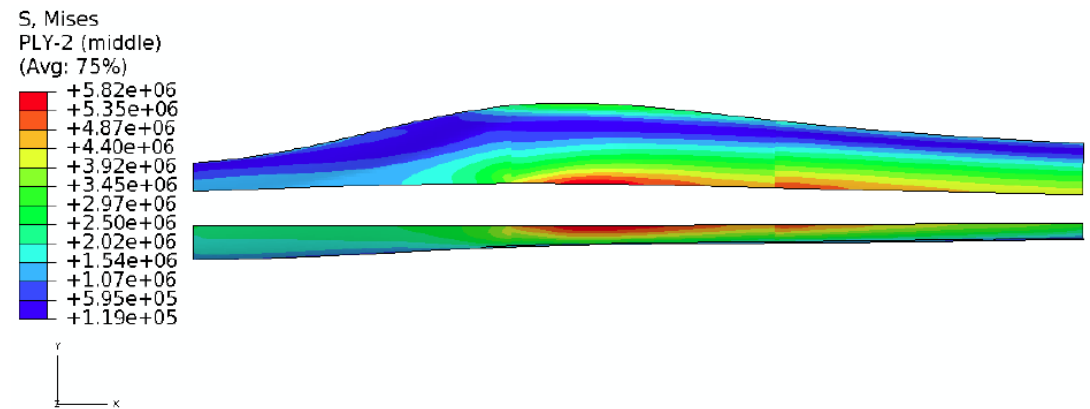

(a)

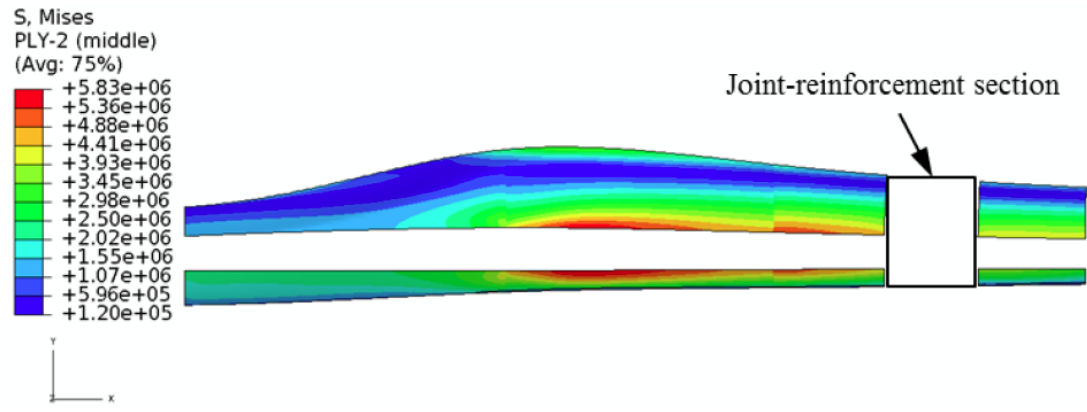

(b)

Figure 10: Von Mises stress in the balsa core of the upper blade skin (5.6 m $<r<46 \mathrm{~m}$ ): (a) SB Model, and (b) MB Model.

Model and MB Model exceeds the allowable strength based on von Mises stress criteria, as seen in Figure 10. In the SB Model, higher von Mises stresses are located at the boundary between the blade skin and the blade skin/spar cap $(20 \mathrm{~m}<r<32 \mathrm{~m})$. This blade skin region has the thicker airfoil, and these dimensions affect the inertia leading to the higher stresses. The maximum von Mises stress experienced by the balsa core of the skin is about $5.82 \mathrm{MPa}$ in compression, which is above its allowable strength of $5.4 \mathrm{MPa}$. Similarly, this damage mode is also observed in the MB Model, and it is not located at all near the jointreinforcement section but at the boundary between the blade skin and the blade skin/spar cap (20 m $<r<32$ $\mathrm{m})$.

\subsection{Required Forces}

Since the cable mechanisms are not physically represented in the simulations, the axial loads are calculated by Equation (10) from the stress fields of MB Model to define and select cables. As seen in Figure 11, the upper and lower Joint-BS/SC regions are in compression and tension, respectively. There coexist both compressive and tensile $S_{11}$ stresses in JointFSW and Joint-ASW regions. Since multiple layers are stacked in the joint region, we look into $S_{11}$ stress distribution through the shell thickness. As an example, let's consider $\mathrm{S}_{11}$ stress distribution through shell thickness in the Joint-BS/SC region as presented in
Figure 12. We can conclude that the maximum values of S11 stress in the lower and upper Joint-BS/SC regions are $7.16 \times 10^{7}$ and $-6.68 \times 10^{7} \mathrm{~N}$, respectively.

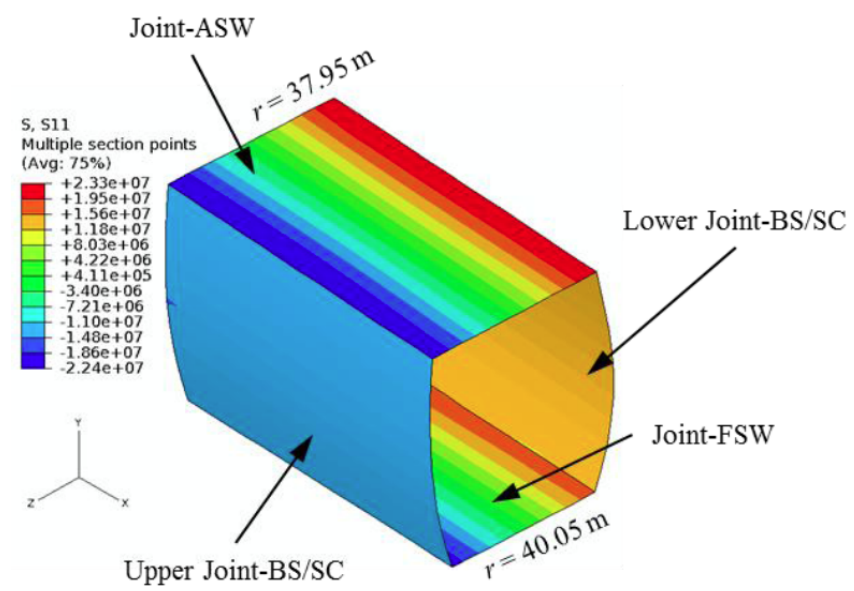

Figure 11: $S_{11}$ stress contour in the outermost layer of the joint region in the MB Model.

The maximum $\mathrm{S}_{11}$ stresses, cross-sectional areas, and axial forces in the joint region are summarized in Table 8. Due to the inherent asymmetry in the blade geometries and applied loads, axial forces for cable mechanisms are not balanced. Since the upper JointBS/SC region experiences compression due to the operational loads, an additional axial force in the upper Joint-BS/SC region is not necessary to produce compression. The absence of the additional compressive force in the upper Joint-BS/SC region 


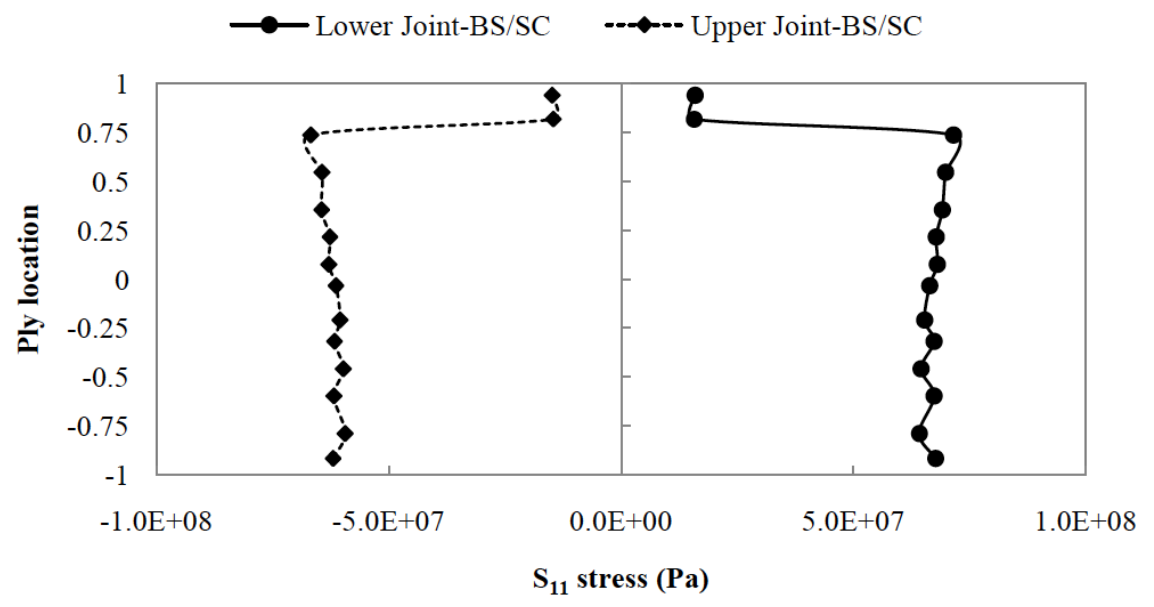

Figure 12: $\mathrm{S}_{11}$ stress distribution through shell thickness in the Joint-BS/SC region.

Table 8: Loads Generated by Cable Mechanisms

\begin{tabular}{|c|c|c|c|}
\hline Location & $\begin{array}{c}\text { Max. } \mathbf{S}_{11} \text { stress } \\
(\mathbf{M P a})\end{array}$ & $\begin{array}{c}\text { Cross sectional area } \\
\left(\mathbf{m}^{2}\right)\end{array}$ & $\begin{array}{c}\text { Axial forces } \\
\mathbf{( N )}\end{array}$ \\
\hline \hline Lower Joint-BS/SC & $7.16 \times 10^{7}$ & $4.87 \times 10^{-2}$ & $3.49 \times 10^{6}$ \\
\hline Upper Joint-BS/SC & $-6.68 \times 10^{7}$ & $4.83 \times 10^{-2}$ & $3.23 \times 10^{6}$ \\
\hline Joint-FSW & $4.00 \times 10^{7}$ & $5.55 \times 10^{-2}$ & $2.22 \times 10^{6}$ \\
\hline Joint-ASW & $5.98 \times 10^{7}$ & $6.42 \times 10^{-2}$ & $3.84 \times 10^{6}$ \\
\hline
\end{tabular}

leads to non-smooth connection of two modules in the joint region. Therefore, the axial force in the upper Joint-BS/SC region should be taken into account when determining the axial force for cable mechanisms.

The total axial force for cable mechanisms is assessed by using this case study and simply given by the sum of four axial forces in the lower Joint-BS/SC, upper Joint-BS/SC, Joint-FSW, and Joint-ASW regions. The total axial force is $12.8 \times 10^{6} \mathrm{~N}$, which is greater than the breaking strength of a traditional commercial cable. Therefore, a single cable with larger diameter or multiple cables are required. Further detailed analysis of introducing axial cable loads to the MB Model confirmed that there are enough compressive forces in the overlap joint region.

Furthermore, the total mass contribution of the cables is calculated assuming a safety factor of 1 for the commercial cables in Table $\mathbf{5}$ and that they extend from the blade root to the tip. The number of cables is simply settled by rounding up the required axial force over breaking strength of a cable. The unit mass for all cables is found from unit mass of a cable multiplied by the number of cables. The total mass of all cables that extend from the blade root to the tip is defined by unit mass for all cables times the blade length. These are summarized in Table 9 illustrating that the additional mass of the stainless cables is in the range of 7.36 7.71 tons. On the other hand, the mass of eleven CFCCs is 1.28 tons that correspond to almost one-sixth of the total mass for the stainless cables. However, these values are still considered relatively heavy in comparison with the mass of the $80 \mathrm{~m}$ blade. Certainly at the detailed design phase considerations, the cable length and locations can be optimized to replace the current simple estimates.

Table 9: Additional Mass to the Blade Due to Stainless Cables and CFCCs

\begin{tabular}{|c|c|c|c|c|}
\hline & $\begin{array}{l}\text { Diameter } \\
\qquad(\mathrm{m})\end{array}$ & \# of cables & $\begin{array}{l}\text { Unit mass for all cables } \\
\qquad(\mathrm{kg} / \mathrm{m})\end{array}$ & Total mass (tons) \\
\hline \multirow{2}{*}{ Stainless cables [34] } & 0.04 & 15 & 96.9 & 7.36 \\
\hline & 0.06 & 7 & 102 & 7.71 \\
\hline CFCCs [35] & 0.04 & 11 & 16.8 & 1.28 \\
\hline
\end{tabular}




\subsection{Natural Frequency}

The undamped natural frequencies of the nonrotating blade, lowest to the sixth, are presented in Table 10. The difference in natural frequencies among the SB Model, MB Model, and MB-IW is at most $1.6 \%$. Since the SB Model shows pure mode shapes in the lower natural frequency, there is no coupling mode of bending and twist together. Similarly, pure mode shapes of the MB Model and MB-IW Model are obtained in this lower frequency range. Therefore, locally increasing stiffness and/or density in the blade does not lead to notable changes in natural frequencies and mode shapes.

Table 10: Undamped Natural Frequencies in $\mathrm{Hz}$

\begin{tabular}{|c|c|c|c|}
\hline Mode & SB Model & MB Model & MB-IW Model \\
\hline \hline 1st flapping & 0.607 & 0.617 & 0.616 \\
\hline 2nd flapping & 1.82 & 1.83 & 1.82 \\
\hline 3rd flapping & 3.84 & 3.90 & 3.88 \\
\hline 1st edge & 0.975 & 0.983 & 0.980 \\
\hline 2nd edge & 3.16 & 3.21 & 3.19 \\
\hline 1st torsional & 4.96 & 5.01 & 4.98 \\
\hline
\end{tabular}

\section{CONCLUSION}

In order to simplify manufacturing, transportation, and assembly requirements, a conceptual joint was proposed to create a modular blade of equal length to a single blade that retained its structural performance. The overall results indicated that such a design is feasible and will sustain operating loads just as well. Herein we highlight the salient knowledge gained from the computational assessments undertaken.

The proposed joint concept for two modular blades to create a full length blade by introducing a jointtransition region did not adversely affect the mechanical and frequency response in comparison to the structural performance of the single continuous blade. The joint-reinforcement section corresponded to $5.5 \%$ of the blade length where both stiffness and density variations were examined to assess the sensitivity to displacement, stress, and frequency responses. It is concluded that the proposed modular composite blade configuration with the cable mechanism is a feasible concept and can be easily adopted in future modular blade designs.

\section{REFERENCES}

[1] United States. US Energy Information Administration. International energy outlook 2016. DOE/EIA-0484. Washington, DC:Department of Energy 2016.

[2] Archer CL, Jacobson MZ. Evaluation of global wind power. $J$ of Geophys Res 2005; 110: D12110.

[3] Japan. Cabinet Secretariat. Final report on the accident at the Fukushima nuclear power stations of Tokyo Electric Power Company. Tokyo: Japan Atomic Energy Agency 2012.

[4] Fukushima Offshore Wind Consortium [Internet]. Fukushima Offshore Wind Consortium; 2014 [cited 2014 September 22]. Available from: http://www.fukushima-forward.jp/english/ index.html

[5] Enercon $\mathrm{GmbH}$. Windblatt: 01/2010. Aurich: Enercon GmbH 2010.

[6] Technical Specification on Wind Turbine SWT-4.0-130 [Internet]. Siemens AG;[cited 2014 March 12]: Available from http://www.energy.siemens.com/us/en/renewableenergy/wind-power/platforms/g4-platform/wind-turbine-swt-40-130.htm\#content=Technical\%20Specification

[7] V164-8.0 MW Breaks World Record for Wind Energy Production [Internet]. MHI Vestas Offshore Wind; [cited 2016 Aug 21]: Available from http://www.mhivestasoffshore.com/ v164-8-0-mw-breaks-world-record-for-wind-energyproduction/

[8] Nanami N. Structural and damage assessment of multisection modular hybrid composite wind turbine blade [Ph.D dissertation].[College Station]:Texas A\&M University 2014.

[9] Nanami N, Ochoa OO. Vibration and dynamic response of hybrid wind turbine blades. In:Liu $D$, editor.Dynamic effects in composites materials.Vol 1.Lancaster: DEStech Publications 2012; pp. 289-301.

[10] Nanami N, Ochoa OO. Bird impact response on a pre-loaded composite wind turbine blade. In: Hoa SV, Hubert P, editors. Proceedings of the 19th International Conference on Composite Materials;2013:Jul 28-Aug 2; Montreal, Canada: Paper ID: 6415 .

[11] Nolet S. Advanced manufacturing initiative final report Presented at the 2014 Wind Turbine Blade Workshop; 2014: Aug 26-28; Albuquerque, New Mexico.

[12] Gamesa 4.5 MW Platform Catalogue. [Online]. Gamesa Corporación Tecnológica; [cited 2013 May 28]: Available fromhttp://www.gamesacorp.com/recursos/doc/productosservicios/aerogeneradores/catalogo-g10x-45mw-eng.pdf

[13] Enercon $\mathrm{GmbH}$. Windblatt:01/2014. Aurich: Enercon $\mathrm{GmbH}$; 2014.

[14] Saenz E, Nuin I, Montejo R, Sanz J. Development and validation of a new joint system for sectional blades. Wind Energy 2014; 18(3): 419-28. http://dx.doi.org/10.1002/we.1704

[15] Wetzel K. Modular blade design \& manufacturing. Presented at the 2014 Wind Turbine Blade Workshop, 2014: Aug 26-28; Albuquerque, New Mexico.

[16] Eyb E, inventor; Eyb E, assignee. Modular rotor blade for a wind turbine and method for assembling same. United States patent US 7654799 B2. 2007 Nov.

[17] Glenn B, Dehlsen JGP, Keller W, Rohm A, Mehrle W, Stuckert M, inventor; Clipper Windpower Technology Inc., assignee. A modular rotor blade for a power-generating turbine and a method for assembling a power-generating turbine with modular rotor blades. International patent WO 2009/090537 A2. 2009 Jul.

[18] Stam R, Starke A, Veldkamp B, Meyer J, inventor; General Electric Company, assignee. Blade modular, a modular rotor blade and a method for assembling a modular rotor blade. United States patent US 8245400 B2. 2012 Aug. 
[19] Somers D. The S816, S817, and S818 Airfoils. Golden: National Renewable Energy Laboratory; 2004. Report No.: NREL/SR-500-36333.

[20] Griffin DA. Blade system design studies volume I: Composite technologies for large wind turbine blades. Albuquerque: Sandia National Laboratories; 2002. Report No.: SAND20021879.

[21] Griffin DA. Blade system design studies volume II: Preliminary blade designs and recommended test matrix. Albuquerque: Sandia National Laboratories; 2004. Report No.: SAND2004-0073.

[22] Burton T, Jenkins N, Sharpe D, Bossanyi E. Wind energy handbook: New York: Wiley 2011. http://dx.doi.org/10.1002/9781119992714

[23] Hau E. Wind turbines: Fundamentals, technologies, application, economics: 2nd ed. New York: Springer-Verlag 2006. http://dx.doi.org/10.1007/3-540-29284-5

[24] Berring P, Branner K, Berggreen C, Knudsen HW. Torsional performance of wind turbine blades-part II: numerical validation. In: Kageyama K, Ishikawa T, Takeda N, Hojo M, Sugimoto $S$, Ogasawara T, editors. Proceedings of the 16th International Conference on Composite Materials 2007: Jul 8-13; Kyoto, Kyoto, Japan.

[25] Laird DL, Montoya FC, Malcolm DJ. Finite element modeling of wind turbine blades. In:Proceedings of 43rd AIAA Aerospace Sciences Meeting and Exhibit 2005: Jan 10-13; Reno, Nevada. http://dx.doi.org/10.2514/6.2005-195

[26] Abaqus Inc. ABAQUS Documentation Collection: Ver. 6.12. Pawtucket: Abaqus Inc. 2012.
[27] Vural M, Ravichandran G. Microstructural aspects and modeling of failure in naturally occurring porous composites. Mech Mater 2003; 35: 523-36. http://dx.doi.org/10.1016/S0167-6636(02)00268-5

[28] Hashin Z, Rotem A. A fatigue failure criterion for fiber reinforced materials. J Compos Mater 1973; 7: 448-64. http://dx.doi.org/10.1177/002199837300700404

[29] Hashin Z. Failure criteria for unidirectional fiber composites. J Appl Mech 1980; 47: 329-34. http://dx.doi.org/10.1115/1.3153664

[30] Kyriazoglou C, Guild FJ. Finite element prediction of damping of composite GFRP and CFRP laminates: a hybrid formulation-vibration damping experiments and Rayleigh damping. Compos Sci Technol 2007; 67: 2643-54. http://dx.doi.org/10.1016/j.compscitech.2004.12.044

[31] Continuous Woven Carbon Fiber. [Online].MatWeb; [cited 2010 Jan 12]: Available fromhttp://www.matweb.com/search/ QuickText.aspx?SearchText=woven\%20carbon

[32] Daniel MD, Ishai O. Engineering mechanics of composite materials. New York: Oxford University Press 1994.

[33] Galvanized and Stainless Aircraft Cable. [Online]. Worldwide Enterprises Inc.; [cited 2013 May 13]: Available from http://www.wwewirerope.com/aircraftcable/

[34] TSK Stainless Ropes. [Online]. Tokyo Rope MFG; [cited 2014 Apr 17]: Available from http://saas.startialab.com/ acti_books/1045176105/5240/_SWF_Window.html

[35] Carbon Fiber Composite Cable. [Online]. Tokyo Rope MFG; [cited 2014 Apr 17]: Available from http://www.tokyorope.co. $\mathrm{jp} /$ product/cfcc/material/index.html

[36] Meier U. Carbon fiber-reinforced polymers: Modern materials in bridge engineering. Struct Eng Int 1992; 2: 7-12. http://dx.doi.org/10.2749/101686692780617020 